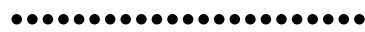

Chirurgie der Leistenhernie 



\section{Chirurgie der Leistenhernie}

\section{Minimalinvasive Operationstechniken}

Herausgeber

Reinhard Bittner Stuttgart

Bernhard J. Leibl Coburg

Martin Ulrich Stuttgart

162 Abbildungen, 128 farbig, 64 Tabellen, 2006 


\section{$\bullet \bullet \bullet \bullet \bullet \bullet \bullet \bullet \bullet \bullet \bullet \bullet \bullet \bullet \bullet \bullet \bullet \bullet \bullet \bullet \bullet \bullet \bullet$}

Bibliographische Information der Deutschen Bibliothek

Die Deutsche Bibliothek verzeichnet diese Publikation in der Deutschen

Nationalbibliografie; detaillierte bibliografische Daten sind im Internet

über < http://dnb.ddb.de > abrufbar.

ISBN 3-8055-7557-2

Dosierungsangaben von Medikamenten

Autoren und Herausgeber haben alle Anstrengungen unternommen, um sicherzustellen, dass die Auswahl und Dosierungsangaben von Medikamenten im vorliegenden Text mit den aktuellen Vorschriften und der Praxis übereinstimmen. Trotzdem muss der Leser im Hinblick auf den Stand der Forschung, Änderung staatlicher Gesetzgebungen und den ununterbrochenen Strom neuer Forschungsergebnisse bezüglich Wirkung und Nebenwirkungen von Medikamenten darauf aufmerksam gemacht werden, dass unbedingt bei jedem Medikament die Packungsbeilage konsultiert werden muss, um mögliche Änderungen im Hinblick auf die Indikation und Dosierung nicht zu übersehen. Gleiches gilt für spezielle Warnungen und Vorsichtsmaßnahmen. Ganz besonders gilt dieser Hinweis für empfohlene neue und/oder nur selten gebrauchte Wirkstoffe.

Alle Rechte vorbehalten. Ohne schriftliche Genehmigung des Verlags dürfen diese Publikation oder Teile daraus nicht in andere Sprachen übersetzt oder in irgendeiner anderen Form mit mechanischen oder elektronischen Mitteln (einschließlich Fotokopie, Tonaufnahme und Mikrokopie) reproduziert oder auf einem Datenträger oder einem Computersystem gespeichert werden.

(C) Copyright 2006 by S. Karger Verlag für Medizin und Naturwissenschaften GmbH,

Postfach D-79095 Freiburg, und S. Karger AG, Postfach CH-4009 Basel

Printed in Germany on acid-free paper by druckhaus köthen $\mathrm{GmbH}$, Köthen/Anhalt

ISBN 3-8055-7557-2 
Inhalt

\section{Vorwort}

Bittner, R. (Stuttgart)

Grundlagen

1 Neuroanatomie der Leistenregion bei besonderer Berücksichtigung endoskopischer Operationstechniken Loeweneck, H. (München)

20 Biomechanik der Bauchdecke unter besonderer Berücksichtigung der Leistenregion

Hollinsky, C.; Sandberg, S. (Wien)

27 Rolle des Ultraschalls in der präoperativen Diagnostik und Hernienklassifikation sowie in der postoperativen Nachsorge Kraft, B. (Nürtingen)

40 Aktuelle Methodenwahl unter spezieller Berücksichtigung der Herniensituation

Kraft, B. (Stuttgart)

58 Indikationen - Kontraindikationen

Jäger, C. (Stuttgart) 
Standardtechniken und perioperatives Management

74 Anästhesiologische Aspekte bei der laparoskopischen Hernioplastik

Junginger, W.; Hafner, C. (Stuttgart)

84 Präoperative Vorbereitung

Ulrich, M. (Stuttgart)

88 Pneumoperitoneum-Technik (Veressnadel, Hasson) und Risiken

Gutt, C.N. (Heidelberg); Schmedt, C.-G. (München)

96 Pathophysiologie des Pneumoperitoneums

Gutt, C.N.; Oniu, T. (Heidelberg)

102 Laparoskopische Leistenhernie:

Geräte, Instrumente und OP-Aufbau

Sedlmayer, F. (Stuttgart)

115 Standardtechnik der transabdominellen präperitonealen Patchplastik (TAPP)

Bittner, R. (Stuttgart)

126 Standard-Technik total extraperitoneale Hernioplastik (TEP)

Tamme, C.; Köckerling, F. (Hannover)

140 Postoperatives Management

Ulrich, M. (Stuttgart)

Ergebnisse bei Primär- und komplexer Hernie, Komplikationsvermeidung

143 Ergebnisse der TAPP bei der Primärhernie Bittner, R. (Stuttgart) 
153 Technische Besonderheiten bei der TAPP-Reparation von Rezidiv-, Skrotal- sowie irreponibler/inkarzerierter Hernie Leibl, B.J. (Coburg)

163 Ergebnisse der Rezidiv- und Skrotalhernienreparation sowie der Therapie von inkarzerierten Hernien Leibl, B.J. (Coburg)

172 Rezidiv nach TAPP, TEP oder konventioneller präperitonealer Netzimplantation - was ist zu tun? Leibl, B.J. (Coburg)

179 Ergebnisse der total extraperitonealen Hernioplastik - TEP

Tamme, C.; Köckerling, F. (Hannover)

188 Praktische Hinweise zur Vermeidung von Komplikationen und Rezidiven bei der TAPP

Kraft, K. (Nürtingen)

205 Praktische Hinweise zur Vermeidung von Komplikationen und Rezidiven bei der TEP

Kuthe, A. (Hannover)

Spezielle Fragestellungen, chronischer Leistenschmerz, evidenzbasierte Ergebnisse

215 Biomaterialien und ihre Gewebekompatibilität Leibl, B.J. (Coburg)

244 TAPP versus TEP aus der Sicht eines TAPP-Spezialisten Kukleta, J.F. (Zürich)

255 TEP versus TAPP aus der Sicht eines TEP-Spezialisten Bailey, M.; Scala, A. (Guildford Surrey)

263 Langzeitergebnisse nach endoskopischer Hernienchirurgie Helbling, C.; Schmid, A.; Schlumpf, R. (Aarau) 
272 Chronische Leistenschmerzen

Vonlanthen, R.; Krähenbühl, L. (Fribourg)

282 Evidenzbasierte Hernienchirurgie - Vergleich endoskopischer Techniken (TAPP/TEP) mit der Shouldice-Reparation und anderen offenen Verfahren ohne Netzimplantation.

Eine Metaanalyse randomisierter (lb) Studien

Bittner, R. (Stuttgart); Sauerland, S. (Köln); Schmedt, C.-G. (München)

301 Evidenzbasierte Hernienchirurgie - Vergleich endoskopischer Techniken (TAPP/TEP) mit der Lichtenstein-Operation und anderen offenen Netzimplantationsverfahren.

Eine Metaanalyse randomisierter (lb) Studien

Schmedt, C.-G. (München); Sauerland, S. (Köln); Bittner, R. (Stuttgart)

322 Kosten

Sauerland, S.; Eypasch, E. (Köln)

329 Ausbildung und Lernkurve

Schmedt, C.-G. (München); Bittner, R. (Stuttgart)

334 Zukunftsperspektiven

Bittner, R. (Stuttgart)

337 Korrespondenzautoren

338 Sachwortverzeichnis 


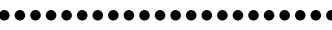 \\ Vorwort}

Bereits vor 10 Jahren haben wir unser erstes Buch zur laparoskopischen Hernioplastik veröffentlicht. Wenngleich einige wesentliche Prinzipien der Operationstechnik auch heute noch unverändert Gültigkeiten haben, so hat sich doch zwischenzeitlich ein riesiger Schatz an neuen Erkenntnissen - sei es die Studienlage, sei es die Implanttechnologie oder die Fixierungsmöglichkeiten betreffend - angesammelt. Es erschien uns daher notwendig, nicht allein das alte Buch mit einigen Aktualisierungen neu aufzulegen, sondern ein vollkommen neues Buch zu schreiben. Es war uns ein Anliegen, auch verstärkt auf die Total Extraperitoneale Patchplastik (TEP) einzugehen, sowie auch auswärtige Autoren mit einzubeziehen. Das Kapitel Anatomie wird jetzt von Herrn Prof. em. H. Loeweneck geschrieben, der hierzu mit seinem Team die weltweit wohl besten Studien durchgeführt hat. Auf die Kapitel Historie und Ergebnisse der offenen Hernienchirurgie wurde verzichtet, dafür neu aufgenommen ein Kapitel zur Biomechanik der Bauchdecke, der Leistungsfähigkeit des Ultraschalls in Diagnostik und Klassifikation der Inguinalhernie, sowie, über die Beschreibung der Standardtechnik TAPP und TEP hinausgehend, ausführliche Beschreibungen des Vorgehens bei der komplizierten Hernie, im Besonderen auch zur Vermeidung von Komplikationen und Rezidiven. Ein besonderes Kapitel ist der laparoskopischen Technik beim Rezidiv nach präperitonealer Netzimplantation gewidmet. Von zwei international anerkannten Experten (Jan Kukleta, Schweiz; Michael Bailey, Großbritannien) werden Vor- und Nachteile der TAPP-Technik versus der TEP abgewogen. Umfangreiche Kapitel beschäftigen sich mit der Biokompatibilität der neuen Netztechnologien, sowie den Schmerzen vor und nach Hernienreparation. Besonders aktuell ist die zusammenfassende Darstellung in Form einer Metaanalyse sämtlicher zur Zeit auswertbarer randomisierter Studien zum Vergleich der laparoskopischen und endoskopischen Technik mit den offenen Nahtverfahren sowie den offenen anterioren Netzimplantationen. Mit reichlich Bildmaterial werden im Besonderen die Einzelheiten der verschiedenen Operationstechniken veranschaulicht.

Zusammenfassend ist es gelungen, ein für jeden Hernienchirurgen attraktives Buch zusammenzustellen, das nicht nur eine Vielzahl von Informationen bietet, sondern auch von praktischem Nutzen bei der täglichen Arbeit ist.

Professor Dr. Dr. h.c. Reinhard Bittner

Klinik für Allgemein- und Viszeralchirurgie, Marienhospital Stuttgart 
